\title{
Enhanced fractional frequency reuse approach for interference mitigation in femtocell networks
}

\author{
Abdullah H. Alquhali ${ }^{1}$, Mardeni Roslee ${ }^{2}$, Mohamed Babikir Abdelgadir ${ }^{3}$, \\ Khaldon Kordi ${ }^{4}$, Khalid S. Mohamed ${ }^{5}$ \\ ${ }^{1,2,4,5}$ Centre for Wireless Technology (CWT), Faculty of Engineering, Multimedia University, Malaysia \\ ${ }^{3}$ Faculty of Engineering, Multimedia University, Malaysia
}

\begin{abstract}
Article Info
Article history:

Received Jan 21, 2020

Revised Mar 5, 2020

Accepted Mar 23, 2020

\section{Keywords:}

Cellular communication

Femtocell networks

Fractional frequency reuse

Interference management

Throughput
\end{abstract}

\begin{abstract}
Small cell networks are expected to heavily be deployed in wireless communication networks due to it ability to enhance signals quality and spectrum utilisation. However, interference is posing a major threat to wireless communication especially cellular femtocell networks whereby its performance is degraded in dense deployment areas. For this reason, an enhanced fractional frequency reuse approach is proposed in this paper to mitigate the interference in femtocell networks. This is achieved by dividing the service area and frequency into three regions and three sets whereby each set is allocated different frequency set. The femtocell location is later obtained and assigned frequency in accordance to the region. The proposed approach helps in reducing the interference, boost the signal to interference plus noise (SINR), and enhance the throughput.
\end{abstract}

This is an open access article under the CC BY-SA license.

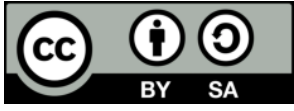

Corresponding Author:

Mardeni Roslee,

Centre for Wireless Technology (CWT),

Faculty of Engineering, Multimedia University,

Cyberjaya 63100, Malaysia.

Email: mardeni.roslee@mmu.edu.my

\section{INTRODUCTION}

Recent technologies rely on wireless communication to enhance the mobility factors and the overall performance because it grants fast delivery rates and financial low-costs. Wireless technology consists of major parts, namely the transmitter and the receiver whereby both communicate through a wireless channel which is related to the communication quality. Subsequently, the technology requires consistent upgrades to assure good quality of communication [1-2]. Different wireless systems adopt different techniques in pursuance of tackling the issues that pose major threats to the capacity and performance of any system [3-4]. For instance, small cells are deployed in cellular communication to solve for capacity and signal quality [5]. However, concerns about interference in femtocell networks were raised due to dense deployment, power management, and resource allocation [6-7]. Interference is often caused by power leaks, double assignment of resources, two or more users exist close to each other etc. This has triggered many researchers to investigate and propose different ways in trials of solving for the raised concerns such as power suppression, resource allocation, frequency partitioning, and interference alignment [8-10].

In view of that, the authors of [11] proposed a femto-aware spectrum arrangement design to circumvent uplink cross-tier interference between a small cell and macrocells by splitting the bandwidth into two segments: shared spectrum between macrocell and femtocell, and the macrocell dedicated spectrum. 
In [12], a scheme is presented to decrease both downlink cross-tier and co-tier interference and boost up the spectral efficiency for orthogonal frequency-division multiple access (OFDMA) based closed access femtocell networks. The frame work uses a composite of clustering of femtocell based on their geographic locations and dynamic frequency band allocation among macrocell and femtocells. The outcome of simulation demonstrated high spectral efficiency as the probability of cross-tier spectrum reuse becomes higher.femtocell networks. The frame work uses a composite of clustering of femtocell based on their geographic locations and dynamic frequency band allocation among macrocell and femtocells. The outcome of simulation demonstrated high spectral efficiency as the probability of cross-tier spectrum reuse becomes higher. The authors of [13] proposed a cross-tier interference reduction scheme based on orthogonal random beamforming in closed-access two-tier femtocell networks. The two factors that affect the macrocell beam subset selection strategy are the intensity of femtocell and the total count of macrocell user equipments (UEs) present in a network. Utilizing the signal to interference plus noise (SINR) data of all the channels evaluated by the macrocell UEs, the macrocell allocates the users and beam subset for each channel by choosing the optimal number of beams adaptively utilizing maximum throughput scheduler at the macrocell. The cross-tier interference is reduced by the number of beams done via adaptive selection, and offers spatial opportunity to small cell to attain the spectrum in an opportunistic approach.

In [14], back-haul or air interface procures scheduling information from the macrocell for both down- link and uplink macrocell UEs. The scheduling information helps dealing with the issue of spectrum sensing which enhances the results so that the resource blocks of distant macrocell UE are employed in the down link and uplink transmission. The results of inter carrier interference (ICI) to femtocell from macrocell UE have also been analysed. The major cause of ICI is the asynchronous arrival of macrocell UE signals at the femtocell. It has been concluded a boost in received ICI power of femtocell is seen when the center frequency is lower. If the cyclic prefix duration is less than the arrival time of the signals of macrocell UEs, a disruption of orthogonality will occur leading to ICI between the sub-carriers.

The authors of [1] proposed an efficient downlink co-tier interference management framework for an OFDMA-based long term evolution (LTE) system in which the information about the path-loss is shared between the femtocells. Furthermore, knowledge regarding the utilization of LTE component carriers is shared with the adjoining femtocells. The femtocell gateway (GW) maintains the inter femtocell coordination messages through S1 connection and is designed to be an intermediate node between mobile core network and femtocells. In [15], the authors tried to mitigate the cross-tier (co-channel) interference between macrocells and femtocells. They utilize frequency reuse coupled with pilot sensing via a frequency sharing structure to achieve the results. When a femtocell is initiated, it detects the pilot signals that the macrocell has sent. After disposing off the sub-band with the greatest received signal power, femtocell utilises the remainder of the frequency sub-bands. Thus, enhancing the SINR for macrocell-UEs as well as the overall network throughput. More details on the previous work concerning the interference management in general are provided in the subsequent section.

\section{RELATED WORKS}

This dynamic power setting can be operated in both reactive and proactive manner, which can be applied to either in closed-loop power setting (CLPS) and open loop power setting mode (OLPS). When the femtocell is in CLPS mode, it modifies its transmission power in a reactive approach. Whereas, when the femtocell is in the OLPS mode, it regulates its transmission power in a proactive approach. Also, while it is switching between the OLPS mode and CLPS mode, the femtocell shifts to a hybrid mode as per the situation of the operation [16]. The authors of [17] proposed an adaptive power management with fractional frequency reuse scheme for co-tier femtocell interference reduction. The scheme readjusts the transmission power of each femtocell by obtaining the distance to other femtocells and estimates the coverage range that will not generate any interference to the neighbouring femtocell. The authors assumed that all femtocells are controlled a central unit that is familiar with the locations of all femtocells in the service area. Although the scheme enhanced the system performance but the paper did not discuss the extent of reducing or increasing the power and how it may affect the network outage.

The work presented in [18], proposed a resource allocation approach for OFDMA based on cognitive femtocells. The aim by using co-tire/cross-tire interference constraints with incomplete channel sensing and QoS to increase the total capacity of all the femtocell users. The minimum and maximum numbers of subchannels engaged by each user are considered to attain the fairness through femtocells.Moreover, power allocation and subchannel algorithm is proposed. The performance of simulations is verified in terms of fairness compared with the existing approach and capacity by using the proposed algorithm. The authors of [19], proposed a semi-static inter-cell interference coordination (ICIC) approach name adaptive frequency reuse (AFR). There are two algorithm within the proposed ARF approach 
which is namely, interference-aware resource allocation and primary subchannel self-configuration (PSC-SC), that served as intracell resource allocation and as ICIC respectively. The proposed AFR approach in terms of spectrum efficiency of cell edge users with a minimum effect in the cell center users of the spectrum efficiency can achieve approximately $100 \%$ gain.

In [20], by using cognitive radio non-orthogonal multiple access (CR-NOMA) for femtocell users the authors proposed a power control and a joint channel allocation scheme. The target is to maximise the throughput of the femtocell users (FUs) with constrained guaranteed QoS. CR-NOMA used at the femto base sation (FBS) through the target to have the guaranteed QoS for FUs. Therefore using pairing, the results in better channel utilisation by reduce the NOMA interference between them. Additionally, to provide for weak users the even/odd number of FUs is differentiated. Numerical results illustrate the improvement of throughput $n$ the proposed approach with providing guaranteed QoS for CR-NOMA based on Femtocell users.

The authors of [21] developed a method to decrease the interference in downlink of FBS considering that the FBSs are diffused in high density based on their old method. The users' devices can receive interference from FBS and reply a warning to all FBSs which can be interfered with it. The power levels were reduced to bring the effect of interference down as soon as the FBSs defined themselves as excessive interference (EI) cells when the number of the received warning excess the number of served user by the cell. The results show that the proposed method is able to increase the throughput by $120 \%$ compared to their previous method, it helps to reduce the power consumption due to the decreased interference in downlink in same spectrumusage.

In [22], the authors proposed a new scheme by using hybrid spectrum allocation where the spectrum divided between the macrocell and femtocells interfering it depending on the demand of their resources, where the far femtocells can share the whole spectrum. The problem here is divided in optimization approach into two sub problems. A low complexity algorithm is proposed to find solution of these sub-problems. The throughput and packet loss were improved in the simulation where a less density femtocell scenario considered. The proposed scheme results in high performance as in the optimal solution obtained by exhaustive search. The authors of [23-25], proposed another interference management frameworks for LTE femtocells that is based on fractional frequency reuse (FFR). The proposition allocates sub-bands to the femtocells from the overall designated frequency band that are being un-utilised in the sub-area of the macrocell. This results in the avoidance of downlink cross tier interference.

The authors of [26] proposed an adaptive FFR infrastructure has been proposed that would reduce downlink interference produced by the femtocells in the neighbourhood of a macrocell to a minimum. The proposed framework makes use of orthogonal FFR radio resource or FFR radio resource hopping allocation based on the highness or lowness of the density as well as the information of the location of the femtocells. The location gives information if it is in an outer region or an inner region. In this paper, an enhanced fractional frequency reuse is proposed whereby the macrocell is divided into three regions in which each region is utilising different frequency spectrum. This implies that the frequency spectrum allocated for the macrocell should be divided into three sets as well. The contributions of this paper are summarised below:

- An enhance fractional frequency reuse mechanism that mitigates the interference exchanged between femtocells and enhance the network performance.

- Better understanding on the interference challenges in femtocell networks and the fractional frequency reuse approach.

- A motivation for further investigation on interference management in dense femtocell deployment. The remainder of the paper is divided as follows: Section 2 provides the literature study, Section 3. presents the system modelling, Section 4 discusses the proposed model, the simulation results are presented in Section 5, and Section 6 concludes the paper.

\section{SYSTEM MODELLING}

In this paper, we initially divide the coverage area into three regions, namely inner, middle, and outer regions. This is because in the conventional FFR scheme, the area is usually divided into two regions. Hence, increasing the regions will for sure enhance the interference mitigation. The frequency is also divided into three sets $f_{1}, f_{2}$, and $f_{S}$. Similarly, this is to assign a frequency set to each region in the service area to prevent interference from occurring in between regions. The frequency assignment is done by extracting the locations of the femtocells within the service area in which all are represented by Cartesian coordinates. We denote the frequency assigned to each femtocell by $\alpha_{s n}$ whereby $n$ is the set identifier. The set of femtocells is referred to as $[|S|, 1,2,3, \ldots, S]$, the set of users is denoted by $[|u|, 1,2,3, \ldots, U]$, and the set of subchannels is denoted by $[|k|, 1,2,3, \ldots, K]$. The group of femtocells in region $n$ is referred to as $S_{n}$. For convenience, we set the radius of each region manually whereas $r_{i}, r_{m}$, and $r_{o}$ denote the inner region radius, 
middle region radius, and the outer region radius, respectively. The simulation is executed in three phases whereby each is referred to as experiment. The experiments setup is presented in Table 1.

Table 1. Experiments setup

\begin{tabular}{|c|c|c|c|}
\hline Experiment & Number of $F_{R A N D}$ & Inner region radius & Middle region radius \\
\hline Experiment 1 & 50 & $\begin{array}{l}33 \% \text { of macrocell base station } \\
\text { (MBS) radius }\end{array}$ & $66 \%$ of MBS radius \\
\hline Experiment 2 & 200 & $33 \%$ of MBS radius & $66 \%$ of MBS radius \\
\hline Experiment 3 & 1000 & $50 \%$ of MBS radius & $75 \%$ of MBS radius \\
\hline
\end{tabular}

\subsection{Problem formulation}

As highlighted earlier, the interference level in any system affects the overall performance. Therefore, it is important to measure the level of interference exchanged received from the environment. This is typically evaluated through the signal to SINR obtained by Equation 1 which is a measure the quality of the received signal in presence of the interference and noise.

$$
S I N R_{s u}=\sum_{k \in K} \frac{P_{s k u} g_{s k u}}{\sum_{i \in S_{n} /\{s\}} P_{i k u} g_{i k u}+N}
$$

whereby $P_{s k u}$ is the transmitted power by $u$ from $s$ at the $k$ th subchannel, $g_{s k u}$ is the channel response, $P_{i k u}$ is the interference, and $N$ is the noise. Thus the problem can be formulated as follows:

$$
\begin{aligned}
& \arg \min \sum_{i \in S_{n} /\{s\}} \sum_{K \in K} P_{s k u} \\
& \text { s.t. } \\
& U_{i}>0 \\
& \forall i \in S_{n} /\{s\}
\end{aligned}
$$

\section{THE PROPOSED MODEL}

When a femtocell is initiated, the location of the femtocell is obtained to calculate the distance $d_{s}$ to the macrocell to identify in which region the femtocell is. Subsequently, the region identifier $\beta_{s n}$ is set according to the following:

$$
\beta=\left\{\begin{array}{lr}
1, & d_{s} \leq r_{i} \\
2, & r_{i}<d_{s} \leq r_{m} \\
3, & d_{s} \geq r_{m}
\end{array}\right.
$$

It can now be said that $\alpha_{s n}$ and $\alpha_{i n} \forall i \in S /\{s\}$ are equal if femtocells $s$ and $i$ are in the same region. Consequently, interference can be received. Additionally, the following algorithm explains how the proposed model operates.

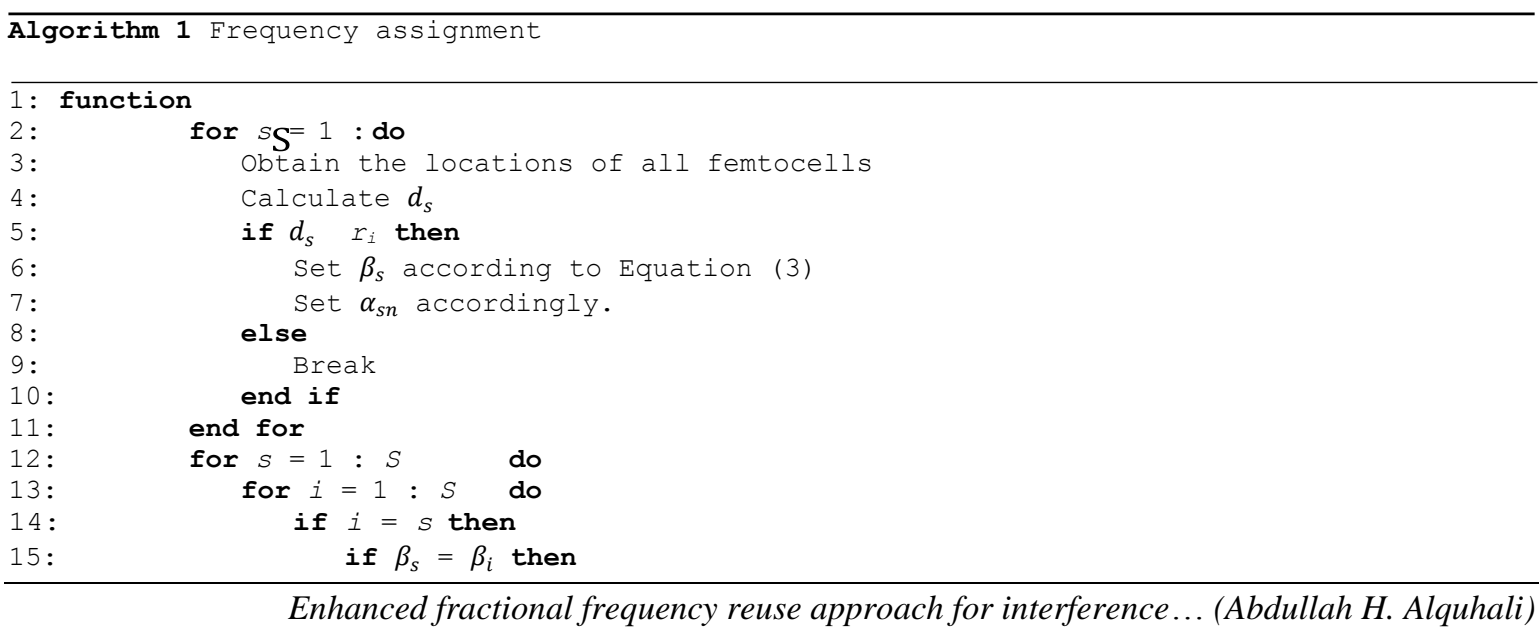




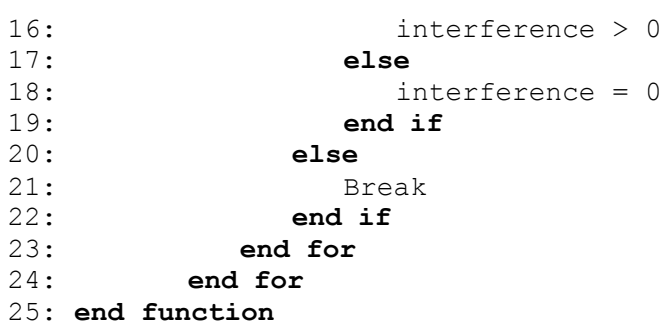

\section{SIMULATION RESULTS}

The simulation results of the proposed scheme are compared to different schemes. The first is when no frequency assignment scheme is considered, and the conventional FFR (2-region) scheme discussed in [26]. The results depicted in Figure 1a depicts that all three users have received approximately $0.46 \mathrm{~mW}$ less interference than the 2-region scheme. This is clearly reflected when measuring both throughput and SINR as in Figure 1b and Figure 1c, respectively. All three users have a maximum of $2.5 \times 10^{8}$ bps increased throughput as compared to the 2-region scheme for all iterations.

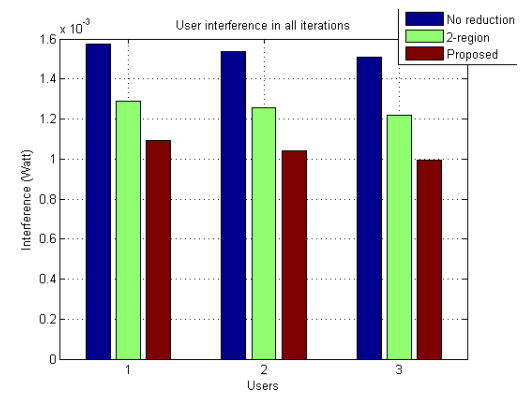

(a) Received interference

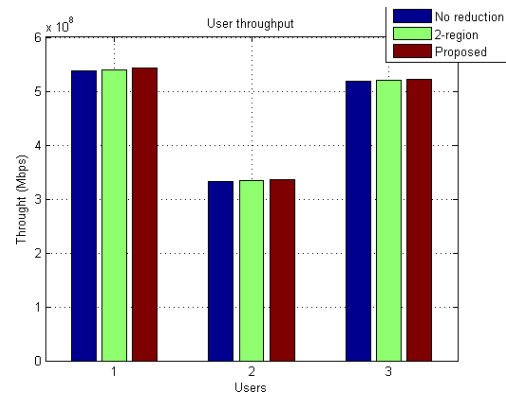

(b) User throughput

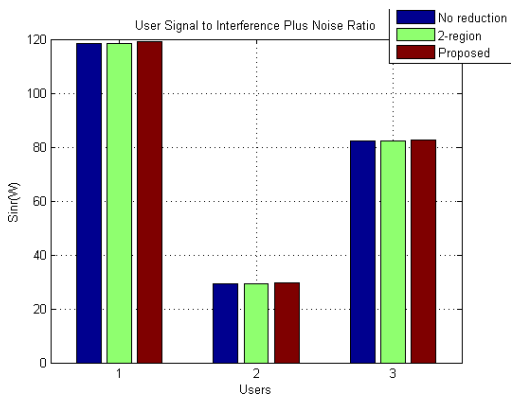

(c) User SINR

Figure 1. Performance evaluation for experiment 1

Furthermore, the results were recorded and displayed in the Figure 2a. This result shows that the all three users have received approximately $0.69 \mathrm{~mW}$ less interference than the 2-region scheme. This result effects the overall throughput and the SINR, as shown in Figure $2 b$ and Figure 2c, respectively. Furthermore, all three users have a maximum of $9.3 \times 10^{8} \mathrm{bps}$ increase in the throughput as compared to the 2-region scheme for all iterations.

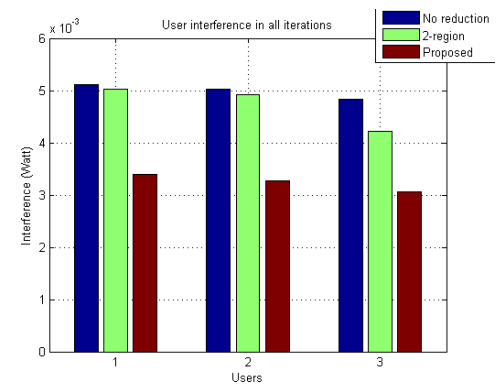

(a) Received interference

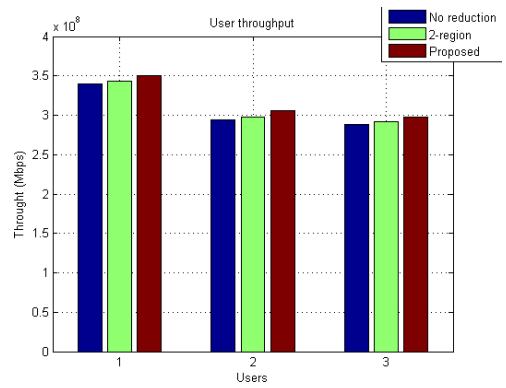

(b) User throughput

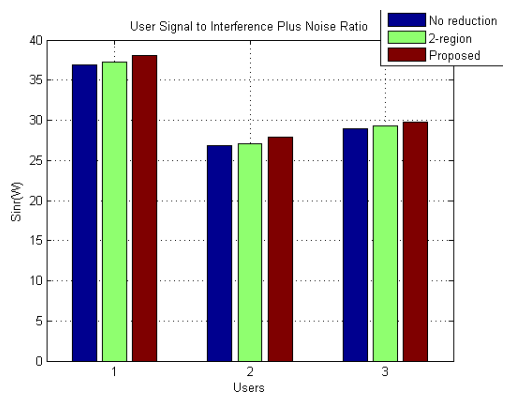

(c) User SINR

Figure 2. Performance evaluation for experiment 2

Moreover, the results shown in Figure 3a shows that all three users have received approximately 0.91 $\mathrm{mW}$ less interference than the 2-region scheme. Therefore, this effects the overall thoughput and the SINR. 
This effects is presented in the bar chart in Figure $3 b$ and Figure 3c, respectively. All three users have a maximum of $4.1 \times 10^{8}$ bps increased throughput as compared to the 2 -region scheme for all iterations.

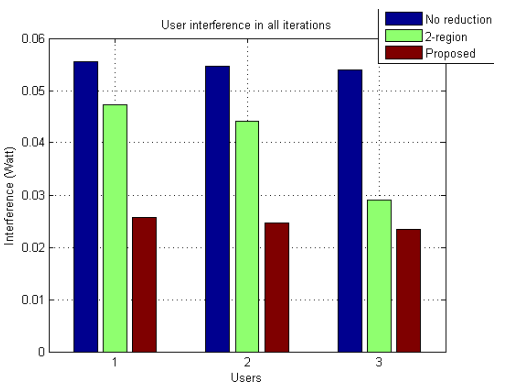

(a) Received interference

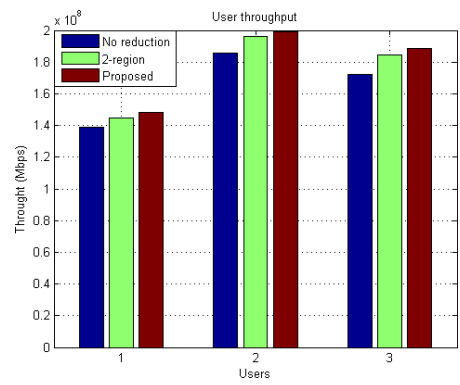

(b) User throughput

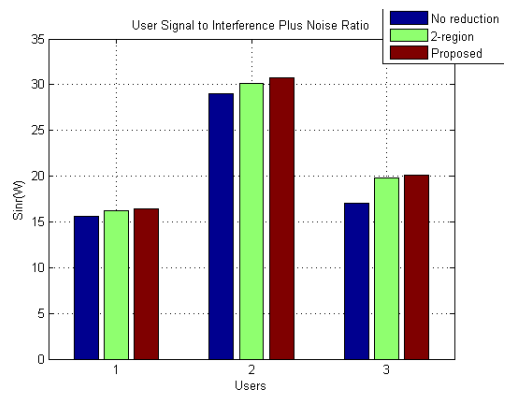

(c) User SINR

Figure 3. Performance evaluation for experiment 3

It can therefore be said that this paper provides a novel and enhanced FFR approach that successfully mitigates the interference and subsequently enhances the network performance. Unlike the literature, the pro- posed scheme relies on dividing the service area into three regions rather than only two in the conventional FFR scheme. It is believed that this is suitable for future communications where interference levels are expected to be high. Similarly, expanding the spectrum might not be feasible due to the expensive spectrum rentals.

\section{CONCLUSION}

In this paper, we propose an approach to mitigate the interference in femtocell networks by dividing the coverage area into three regions rather than two in the fractional frequency reuse approach. The proposed algorithm has minimised the interference, enhanced the SINR, and improved the throughput of the system as compared to the previous approaches that were taken from the literature. In addition, the approach considered in this paper can also be used in dense networks which promotes it for further consideration in future networks. The future directions of this work is to increase the number of users and employ localisation techniques whereby the performance of the system is tested under localisation acquisition errors.

\section{REFERENCES}

[1] G. Miao, J. Zander, K. W. Sung, and S. B. Slimane, "Fundamentals of mobile data networks," Cambridge University Press, 2016.

[2] P. Manhas and M. Soni, "Optimized ofdm model using cma channel equalization for ber evaluation," Bulletin of Electrical Engineering and Informatics, vol. 6, no. 2, pp. 133-139, 2017.

[3] S. Suherman, "Wifi-friendly building to enable wifi signal indoor," Bulletin of Electrical Engineering and Informatics, vol. 7, no. 2, pp. 264-271, 2018.

[4] S. Guruacharya, D. Niyato, E. Hossain, and D. I. Kim, "Hierarchical competition in femtocell-based cellular networks," in 2010 IEEE Global Telecommunications Conference GLOBECOM 2010. IEEE, pp. 1-5, 2010.

[5] K. S. Mohamed, M. Y. Alias, and M. Roslee, "Review on femto-cell networks interference management techniques," International jouranl of engineering and technology, vol. 10, no. 4, pp. 1248-1262, 2018.

[6] A. H. Alquhali, M. Roslee, M. Y. Alias, and K. S. Mohamed, "Survey: D2d communication for spectral efficiency improvement and interference reduction," Bulletin of Electrical Engineering and Informatics, vol. 9, no. 3, pp. 1085-1094, 2020.

[7] K. S. Mohamed and M. Y. Alias, "An efficient femto-cell channel assignment scheme," in lEICE Infor-mation and Communication Technology Forum, 2015.

[8] V.-G. Nguyen, T.-X. Do, and Y. Kim, "Sdn and virtualization-based lte mobile network architectures: A comprehensive survey," Wireless Personal Communications, vol. 86, no. 3, pp. 1401-1438, 2016.

[9] M. Guowang, "Fundamentals of mobile data networks," 2016.

[10] A. Alhammadi, M. Roslee, M. Y. Alias, I. Shayea, S. Alraih, and K. S. Mohamed, "Auto tuning self- optimization algorithm for mobility management in lte-a and 5g hetnets," IEEE Access, vol. 8, pp. 294-304, 2020.

[11] Y. Wu, D. Zhang, H. Jiang, and Y. Wu, "A novel spectrum arrangement scheme for femto cell deployment in lte macro cells," in 2009 IEEE 20th International Symposium on Personal, Indoor and Mobile Radio Communications. IEEE, pp. 6-11, 2009. 
[12] D. López-Pérez, A. Valcarce, G. De La Roche, and J. Zhang, "Ofdma femtocells: A roadmap on interfer- ence avoidance," IEEE Communications Magazine, vol. 47, no. 9, pp. 41-48, 2009.

[13] S. Park, W. Seo, Y. Kim, S. Lim, and D. Hong, "Beam subset selection strategy for interference reduction in two-tier femtocell networks," IEEE Transactions on Wireless Communications, vol. 9, no. 11, pp. 3440-3449, 2010.

[14] T.-H. Kim and T.-J. Lee, "Throughput enhancement of macro and femto networks by frequency reuse and pilot sensing," in 2008 IEEE International Performance, Computing and Communications Conference. IEEE, pp. 390-394, 2008.

[15] N. Saquib, E. Hossain, L. B. Le, and D. I. Kim, "Interference management in ofdma femtocell networks: Issues and approaches," IEEE Wireless Communications, vol. 19, no. 3, pp. 86-95, 2012.

[16] R. Zhang, Y.-C. Liang, and S. Cui, "Dynamic resource allocation in cognitive radio networks," IEEE Signal Processing Magazine, vol. 27, no. 3, pp. 102-114, 2010.

[17] K. Sheikhidris, M. Y. Alias, and M. Roslee, "Adaptive power management with fractional frequency reuse scheme for co-tier femto-cell interference reduction," in 2017 IEEE 13th Malaysia International Conference on Communications (MICC). IEEE, pp. 311-315, 2017.

[18] H. Zhang, C. Jiang, X. Mao, and H.-H. Chen, "Interference-limited resource optimization in cognitive femtocells with fairness and imperfect spectrum sensing," IEEE Transactions on Vehicular Technology, vol. 65, no. 3, pp. 1761-1771, 2015.

[19] G. Huang and J. Li, "Interference mitigation for femtocell networks via adaptive frequency reuse," IEEE Transactions on Vehicular Technology, vol. 65, no. 4, pp. 2413-2423, 2015.

[20] I. Budhiraja, S. Tyagi, S. Tanwar, N. Kumar, and M. Guizani, "Cross layer noma interference mitigation for femtocell users in 5g environment," IEEE Transactions on Vehicular Technology, vol. 68, no. 5, pp. 4721-4733, 2019.

[21] K.-C. Ting, C.-C. Tseng, H.-C. Wang, and F.-C. Kuo, "Advanced interference aware power control (aiapc) scheme design for the interference mitigation of femtocell co-tier downlink in lte/lte-a and the future $5 \mathrm{~g}$ networks," in Information Systems Design and Intelligent Applications. Springer, pp. 548-559, 2018.

[22] Y. L. Lee, J. Loo, T. C. Chuah, and A. A. El-Saleh, "Fair resource allocation with interference mitigation and resource reuse for lte/lte-a femtocell networks," IEEE Transactions on Vehicular Technology, vol. 65, no. 10, pp. 8203-8217, 2016.

[23] J. G. Andrews, R. K. Ganti, M. Haenggi, N. Jindal, and S. Weber, "A primer on spatial modeling and analysis in wireless networks," IEEE Communications Magazine, vol. 48, no. 11, pp. 156-163, 2010.

[24] T. Adiono, S. F. Anindya, S. Fuada, and M. Y. Fathany, "Curtain control systems development on mesh wireless network of the smart home," Bulletin of Electrical Engineering and Informatics, vol. 7, no. 4, pp. 615-625, 2018.

[25] S. Su and S. Wang, "A simple monitoring network system of wireless sensor network," Bulletin of Elec- trical Engineering and Informatics, vol. 1, no. 4, pp. 251-254, 2012.

[26] J. H. Lim, R. Badlishah, and M. Jusoh, "Lte-fractional frequency reuse (ffr) optimization with femtocell network," in 2014 2nd International Conference on Electronic Design (ICED). IEEE, pp. 527-532, 2014.

\section{BIOGRAPHIES OF AUTHORS}
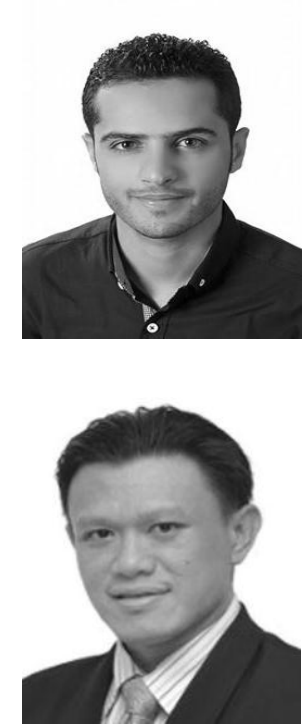

Abdullah H. Alquhali received the B.Eng degree in electrical and communications engineering from Sana'a University in 2012 and Master of Engineering in telecommunications from Multimedia Uni- versity (Malaysia) in 2018. He is currently doing his Ph.D. degree in Efficient Spectrum Utilization Algorithm Based on Distance of 4G/5G D2D Wireless Technologies. His current research interests include $4 \mathrm{G} / 5 \mathrm{G}$, resource allocation, spectrum efficiency and Device-to-Device communications, and next generation cellular networks. Further info on his homepage: http://diagnostics.my/imamul

Mardeni Roslee is a registered Chartered Engineer with the Engineering Council United Kingdom, and Member with The Institution of Engineering and Technology (IET), United Kingdom. As a Chartered Engineer, he bring a diversified range of engineering experience in design \& development and engineering management. His experiences include the consultation, professional institution and academic sectors. He is a Senior Member of IEEE and senior member of IACSIT. His current research interests are wireless mobile communication and radar communication system. 

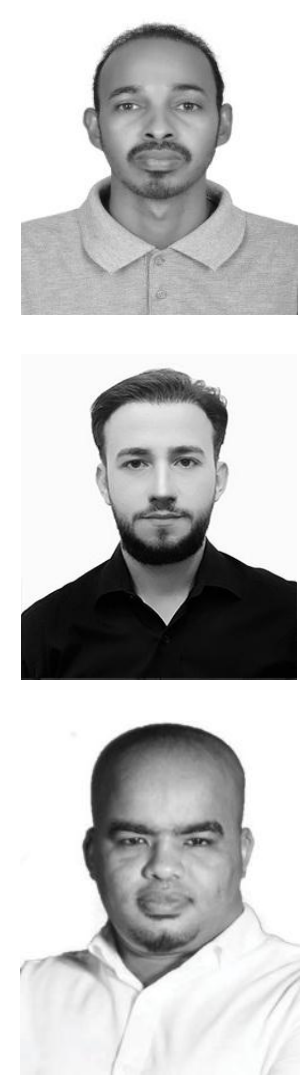

Mohamed Babikir Abdulgadir received his Bacheloer degree in Telecommunication engineering from Mashreq University, Sudan in 2017, and his Master in Engineering of Telecommunications degree from Multimedia University (Malaysia) in 2019. His research interests include Femto cell in 4G-5G system and mitigation of interferences.

Khaldon Kordi received his Bachelor of Science degree in electrical and electronic engineering from Near East University (Cyprus) in 2016, and Master. Eng. Telecommunications degree fr om Multimedia University (Malaysia) in 2018. He is doing his PhD in Multimedia University. His research interests include $5 \mathrm{G}$ channel modeling and Millimeter wave.

Khalid S. Mohamad received his Bachelor in Telecommunication engineering from Future Univer- sity, Sudan 2011. He then received his Master of Engineering in Telecommunication from Multime- dia University, Malaysia 2014. Currently, he is pursuing Ph.D. of engineering in Telecommunication at Multimedia University, Malaysia. He is also registered in Board of Engineers Malaysia (BEM) as a graduate engineer since 9th April, 2019. His research interests include cellular communication, 4G, 5G, IoT, beamforming, MIMO, and interference management in heterogeneous networks. 